Archive for

Organic Chemistry

Arkivoc 2020, part viii, 12-19

\title{
Diels-Alder reactions with 1,1-diethoxybut-3-yn-2-one and some 1,1-diethoxy-5- hydroxyalk-3-yn-2-ones and their acetates
}

\author{
Frank O. Hansen and Leiv K. Sydnes* \\ Department of Chemistry, University of Bergen, Allégt. 41, NO-5007 Bergen, Norway \\ Email: leiv.sydnes@uib.no
}

Received 08-13-2020

Accepted 09-15-2020

Published on line $10-05-2020$

\section{Abstract}

The title compounds were reacted with a few conjugated dienes at room temperature and above. The alcohols were unreactive, but the other ynones reacted at a reasonable rate. Conceivably, the expected cyclohexa-1,4-diene adducts were formed, but they were unstable and aromatized to the corresponding benzene derivatives, which were isolated in low to excellent yield.

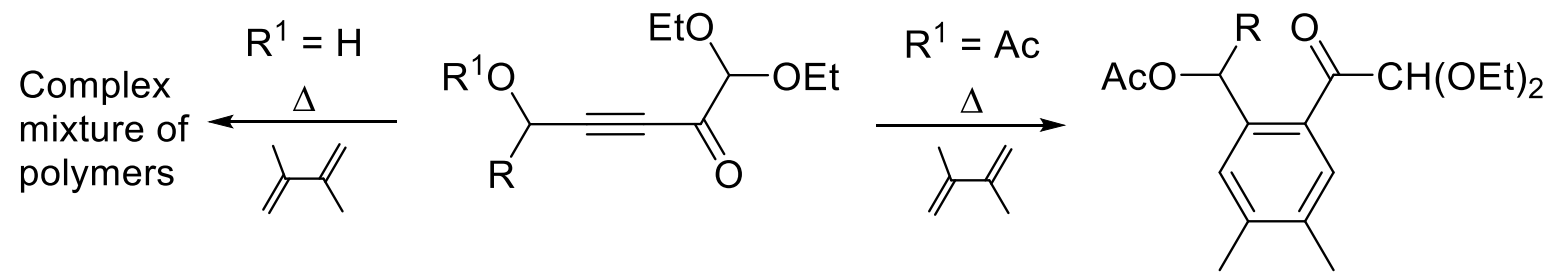

Keywords: Conjugated ynones, propargylic alcohols, propargyl acetates, 1:1 adducts, aromatization 


\section{Introduction}

The Diels-Alder reaction is a valuable transformation in organic synthesis. There are two main versions of the reaction, but the most common by far involves the addition of an electron-rich $4 \pi$ conjugated diene to an electron-poor $2 \pi$ partner, the so-called dienophile. Most dienophiles are alkenes attached to one or several electron-withdrawing groups, but some conjugated ynones have also been utilized. Reviews of a series of basic studies involving $\alpha, \beta$-unsaturated alkynones have been published $^{1-3}$ and so have applications of such dienophiles in natural-product synthesis. ${ }^{4-6}$ The results presented in these publications have been applied and developed further in recent years and made Diels-Alder reactions with ynones an attractive tool in organic synthesis. $^{7}$

The structural variation among the ynones used as dienophiles is rather small. The substituents close to the ynone moiety are limited to a selection of aryl groups, some alkyl groups, and the trimethylsilyl moiety, ${ }^{7}$ and our easy access to conjugated alkynones of the alk-3-yn-2-one type, ${ }^{8-10}$ with polar substituents attached to $\mathrm{C}-1$ and $\mathrm{C}-5$, therefore encouraged us to investigate their reaction with some conjugated dienes commonly used in Diels-Alder reactions. The results of these studies are reported here.

\section{Results and Discussion}

An ynone used relatively much in Diels-Alder reactions is but-3-yn-2-one. ${ }^{1-3}$ It was therefore natural to pick 1,1-diethoxybut-3-yn-2-one (1), easily available in high yield from ethyl vinyl ether, ${ }^{9.10}$ as the first dienophile to get an impression of the influence of polar substituents. In order to estimate the reactivity, exploratory experiments were carried out with isoprene varying the solvent, reaction temperature, and reaction time. $A$ general picture emerged: 1) diluted solutions stirred at room temperature gave no product(s) and the reactants were recovered almost quantitatively; 2) reactions performed in a surplus of diene at reflux for 2-3 days afforded one or two products and a variable amount of a very viscous material, probably a mixture of polydiene on the basis of IR and NMR spectra. ${ }^{11}$ Based on these observations, ynone 1 was reacted with five dienes (2) by heating mixtures of 1 and a 10 times molar excess of the diene at reflux. Two dienes, 4-methyl1,3-pentadiene and 2,5-dimethyl-2,4-hexadiene, both with geminal methyl groups, did not react. The three remaining dienes, 2a-2c, however, gave a 2,2-diethoxyacetylated methylated benzene derivative (3) along with fair amounts of polydiene, and $\mathbf{2} \mathbf{a}$ and $\mathbf{2} \mathbf{b}$ furnished in addition a minor amount of the benzoic acid (4) corresponding to $\mathbf{3 a}$ and $\mathbf{3 b}$, respectively (Scheme 1). The invoked primary products, the 1-diethoxyacetyl-1,4cyclohexadiene analogues to $\mathbf{3}$, were not detected in any case; they are, not unexpectedly unstable and aromatized quickly, conceivably by reaction with oxygen. ${ }^{12-14}$ The by-product in this oxidation is water, which reacts with $\mathbf{3}$ to form benzoic acids $\mathbf{4}$ and formaldehyde diethyl acetal.

The results summarized in Scheme 1 clearly show that the adduct formation from 1 is substrate dependent. Thus, the reactivity of 1 in the Diels-Alder reaction deviates from that of the ethoxy-free analogue but-3-yn-2-one with respect to both relative yield ${ }^{12}$ and selectivity. ${ }^{13}$ This difference can be understood in terms of a combination of the dienophiles' electron density and the steric hindrance at the diene termini. The isopropenyl terminal carbon is the most electron rich in the dienes and should therefore become attached to the $\beta$ carbon of ynone $\mathbf{1}$. Formation of only ketone $\mathbf{3}$ proves that this mode of attack occurred consistently; the adduct formation was therefore regiospecific. But unlike $\mathbf{2 a}$ and $\mathbf{2} \mathbf{b}, \mathbf{2 c}$ is methylated at the other terminus and that would make its bond formation at the ynone $\alpha$ carbon less favourable for steric reasons. Thus, a lower yield when $\mathbf{2 c}$ is applied is expected, and that was indeed observed (Scheme 1 ). 


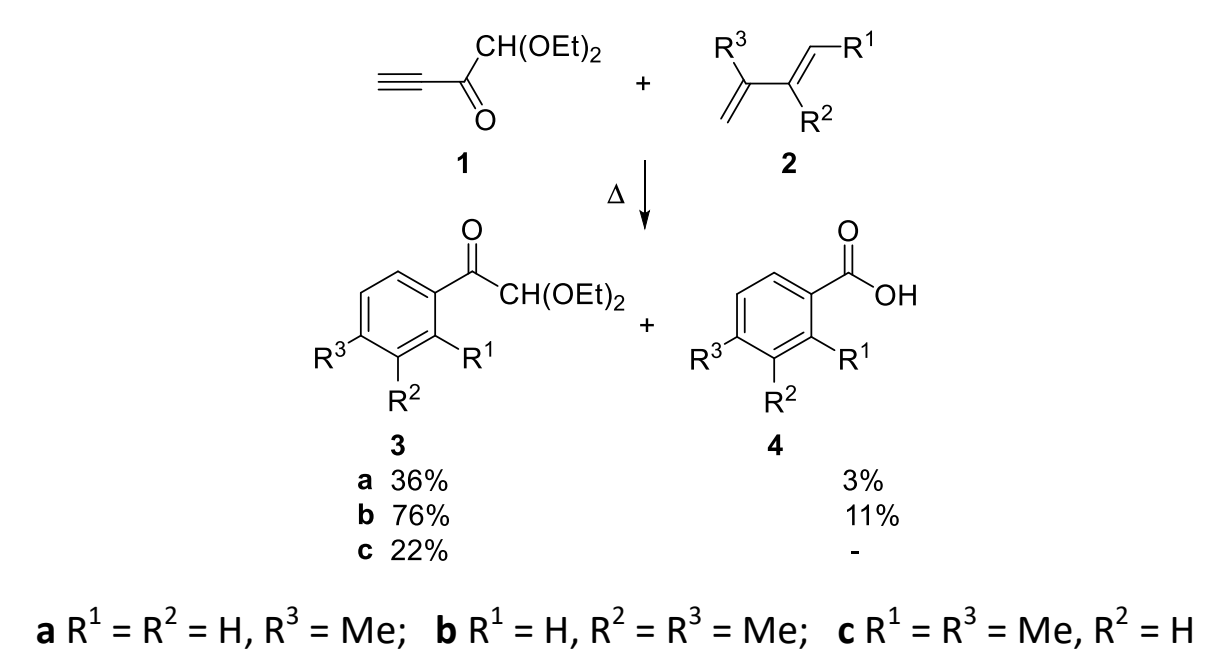

\section{Scheme 1.}

Some chain-elongated derivatives of 1 with a hydroxyl group $\delta$ to the carbonyl group were then used as dienophiles in reactions with a number of dienes including the two dienes that gave the best yields with 1 , viz. $\mathbf{2 a}$ and $\mathbf{2 b}$. Four 1,1-diethoxy-5-hydroxyalk-3-yn-2-ones $(\mathbf{5})^{15,16}$ were reacted, but surprisingly, cycloadduct formation was not observed in a single case. No other products from $\mathbf{5}$ were detected either; the only reaction that occurred was polymerization of the dienes. ${ }^{11,17,18}$

Finally, the acetates of alcohols 5, 5,5-diethoxy-4-oxo-1-R-alk-2-ynyl acetate (6a-6d), ${ }^{15,16}$ were reacted with 2,3-dimethylbuta-1,3-diene (2b), the diene that gave the best results with ynone $\mathbf{1}$. Unlike alcohols $\mathbf{5}$, all the esters underwent the Diels-Alder reaction and gave one product only, benzene derivate 7 (Scheme 2), which conceivably originated from oxygen-induced dehydrogenation of the primary 1,4-cycloheaxdiene DielsAlder adduct. ${ }^{14}$ The yield varied from medium to excellent (41-90\%) (Scheme 2). The reason for this variation is not clear, but since the electronic impact on the ynone moiety does not vary much, the steric crowding around the $\beta$ carbon probably plays the most important role. Polymer material was neither isolated nor spotted, but such a product may very well have been invisible and subsequently attached to the silica gel during the flash-chromatography work-up and therefore avoided detection.

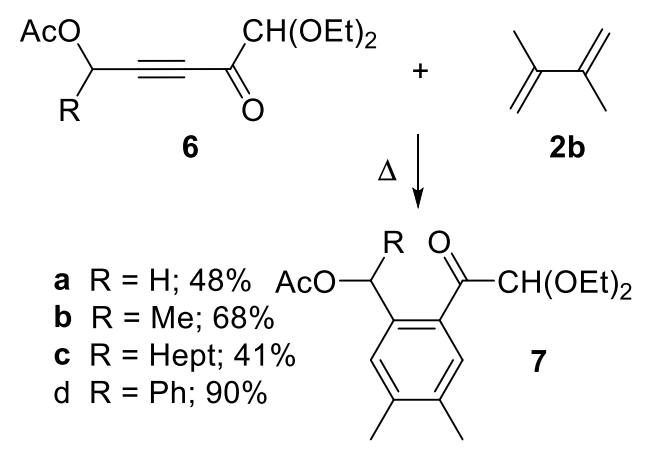

Scheme 2. The Diels-Alder reaction between 2,3-dimethylbuta-1,3-diene (2b) and 5,5-diethoxy-4-oxo-1-R-alk2-ynyl acetate (7). 
The results presented here show that the reactivity of the ynones is somewhat influenced by the substituent(s) attached to the alkynone chain near the $\alpha, \beta$-unsaturated ynone moiety. Since the reaction conditions required for 1 and 6 to react are comparable to those used when but-3-yn-2-one is reacted with the same dienes, the electronic impact from the ethoxy and acetyloxy groups is judged to rather unimportant compared to the steric influence. This conclusion is supported by the yields of $\mathbf{3}$ and $\mathbf{7}$, which can be rationalized in terms of variation in steric congestion around the ynone section of the dienophiles. When propargylic alcohols $\mathbf{5}$ are reacted, however, additional interactions become significant and prevent cycloaddition from taking place. Two conceivable effects can be envisaged to be involved. By hydrogen bonding to the carbon-carbon, triple-bond electron cloud, ${ }^{19-21}$ the $\mathrm{OH}$ group is pulled slightly closer to the triple bond, and this may facilitate a Michael-like interaction between the oxygen atom and the $\beta$ carbon. ${ }^{22}$ As a result, the electrophilicity and availability of the $\beta$ carbon are reduced, apparently enough to make cycloaddition so unfavorable that the reaction does not occur.

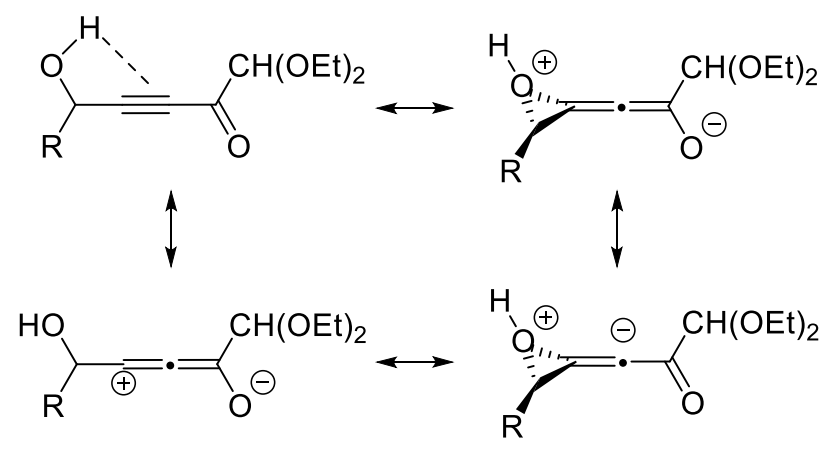

Scheme 3. Some contributing forms describing the interaction between the propargylic OH group and the $\mathrm{C}-\mathrm{C}$ triple bond.

\section{Conclusions}

In summary, ethoxy and acetyloxy groups in the vicinity of the $\alpha, \beta$-unsaturated ynone moiety in conjugated alkynones influence the reactivity in Diels-Alder reactions sterically only. However, a hydroxyl group in $\gamma$ position renders the ynones unreactive towards 1,3-butadienes, which instead suffer polymerization.

\section{Experimental Section}

General. The Diels-Alder reactions were performed under dry conditions in the presence of air. IR spectra were recorded on a Nicolet Impact 410 infrared spectrophotometer; the intensity of the absorptions is characterized as weak (w), medium (m), and strong (s). NMR spectra were run on a Bruker Spectrospin AC 200 F or a Bruker Spectrospin DMX 400. Chemical shifts are reported downfield from TMS, the multiplicity is given as s (singlet), d (doublet), $t$ (triplet), q (quartet), $m$ (multiplet), and dd (doublet of doublet), and coupling constants are given in Hz. GC analyses were performed on a HP 5890 Gas Chromatograph with a flame ionization detector and a HP Ultra 1 column (100\% dimethylpolysiloxane, 25 m, $0.2 \mathrm{~mm}$ i.d., $0.33 \mu \mathrm{m}$ ). Flash chromatography (FC) was performed with Silica gel (230-400 mesh) as the stationary phase and mixtures of hexane and ethyl acetate as the mobile phase. The composition of the mobile phase is described in the 
Supplementary Material. Thin-layer chromatographic (TLC) analyses were carried out with Silica gel (60 $\left.F_{254}\right)$ on aluminium sheets with mixtures of hexane and ethyl acetate as the mobile phase. Visualization of the chromatograms was carried out with phosphomolybdic acid $\left(\mathrm{H}_{3}\left[\mathrm{P}\left(\mathrm{Mo}_{3} \mathrm{O}_{10}\right)_{4}\right]\right)$ in ethanol followed by heating. Mass spectra were obtained on a JEOL AccuTOF T100GC spectrometer. The instruments were operated in the DART/ESI+ mode at 10-15 eV.

Chemicals. 3,3,4,4-Tetraethoxybut-1-yne, synthesized from ethyl vinyl ether as described in the literature, ${ }^{9,10}$ was used to prepare 1,1-diethoxybut-3-yn-2-one $(\mathbf{1})^{10}$ and 1,1-diethoxy-5-hydroxyalk-3-yn-2-ones (5). ${ }^{15}$ Hydroxyketones 5 were then used to synthesize 5,5-diethoxy-4-oxo-1-R-alk-2-ynyl acetates $6 \mathbf{6}-6 \mathbf{d}$. ${ }^{15,16}$ All chemicals used to perform these preparations and the reactions described below were commercially available from Sigma-Aldrich Chemical Company and used without further purification.

Reaction of ynone 1 with diene 2; general procedure. 1,1-Diethoxybut-3-yn-2-one $(0.47 \mathrm{~g}, 3.0 \mathrm{mmol})$ was mixed with the diene $(30 \mathrm{mmol}$ ) and stirred under reflux in a round-bottom flask under air for 48-72 hr. The product mixture was then filtered to isolate crystalline material formed, which was recrystallized from dichloromethane. TLC analysis indicated the presence of one product in the filtrate, and this was isolated pure by FC, eluting with a gradient of hexanes and ethyl acetate as described in the Supplementary Material.

Ynone 1 was reacted with five dienes, of which three gave products.

Reaction with isoprene (2a). The product isolated by FC was 2,2-diethoxy-1-(4-tolyl)ethanone (3a) (colourless liquid, $0.24 \mathrm{~g}, 36 \%)$. IR (ATR, cm $\left.{ }^{-1}\right): 2977(\mathrm{w}), 2880(\mathrm{w}), 1685(\mathrm{~m}), 1606(\mathrm{~m}), 1444(\mathrm{w}), 1373(\mathrm{w}), 1289(\mathrm{~m}), 1112$ $\left.(\mathrm{m}), 1054(\mathrm{~s}), 905(\mathrm{w}), 830(\mathrm{w}), 814(\mathrm{w}), 787(\mathrm{w}), 746(\mathrm{~m}), 711(\mathrm{w}), 685(\mathrm{w}), 654(\mathrm{w}) .{ }^{1} \mathrm{H} \mathrm{NMR}\left(500 \mathrm{MHz}^{\mathrm{CDCl}}\right)_{3}\right):$ $\delta_{\mathrm{H}} 8.08\left(2 \mathrm{H}, \mathrm{d},{ }^{3} \mathrm{~J} 8.4,2 \mathrm{CH}\right.$ aromatic), $7.27(2 \mathrm{H}, \mathrm{m}, 2 \mathrm{CH}$ aromatic), $5.29(1 \mathrm{H}, \mathrm{s}, \mathrm{CH}$ methine), 3.79-3.60 $(4 \mathrm{H}, \mathrm{m}$, $2 \mathrm{CH}_{2}$ ethoxy), $2.41\left(3 \mathrm{H}, \mathrm{s}, \mathrm{CH}_{3}\right.$ tolyl), $1.25\left(6 \mathrm{H}, \mathrm{t},{ }^{3} \mathrm{~J} 7.1,2 \mathrm{CH}_{3}\right.$ ethoxy). ${ }^{13} \mathrm{C} \mathrm{NMR}\left(100 \mathrm{MHz}, \mathrm{CDCl}_{3}\right): \delta_{\mathrm{C}} 194.4$ (1C=O ketone), 145.0 (1C aromatic), 131.9 (1C aromatic), 130.5 (2CH aromatic), 129.7 (2CH aromatic), 102.9 (1CH methine), 63.7 ( $2 \mathrm{CH}_{2}$ ethoxy), 22.4 ( $1 \mathrm{CH}_{3}$ tolyl), $15.9\left(2 \mathrm{CH}_{3}\right.$ ethoxy). HRMS: $\mathrm{m} / z$ calcd for $\mathrm{C}_{11} \mathrm{H}_{13} \mathrm{O}_{2}[\mathrm{M}-$ $\mathrm{OEt}]^{+} 177.09155$, found 177.09161 . The crystalline material was 4-methylbenzoic acid (4a); $10 \mathrm{mg}(3 \%) ; \mathrm{mp}$ $176-179{ }^{\circ} \mathrm{C}$ (lit. $\left.{ }^{23} 177-180{ }^{\circ} \mathrm{C}\right)$.

Reaction with 2,3-dimethylbuta-1,3-diene (2b). The product isolated by FC was 2,2-diethoxy-1-(3,4-dimethylphenyl)ethanone (3b) (colourless oil, $0.25 \mathrm{~g}, 36 \%)$. IR (ATR, cm $\left.{ }^{-1}\right): 2976(\mathrm{~m}), 2877(\mathrm{w}), 1682(\mathrm{~m}), 1606(\mathrm{~m})$, 1570 (w), 1447 (w), 1373 (w), 1324 (w), 1302 (w), 1274 (w), 1247 (w), 1109 (s), 1055 (s), 1022 (s), 938 (w), 908 (w), 802 (w), 748 (w), 709 (w), 643 (w). ${ }^{1} \mathrm{H}$ NMR (400 MHz, CDCl $)_{3}$ : $\delta_{\mathrm{H}}$ 7.90-7.93 (2H, m, 2CH aromatic), 7.21 $\left(1 \mathrm{H}, \mathrm{d},{ }^{3} \mathrm{~J} 8.5,1 \mathrm{CH}\right.$ aromatic), $5.31\left(1 \mathrm{H}, \mathrm{s}, \mathrm{CH}\right.$ methine), 3.80-3.58 $\left(4 \mathrm{H}, \mathrm{m}, 2 \mathrm{CH}_{2}\right.$ ethoxy), $2.32\left(6 \mathrm{H}, \mathrm{s}, 2 \mathrm{CH}_{3}\right.$ aryl), $1.25\left(6 \mathrm{H}, \mathrm{t},{ }^{3} \mathrm{~J} 7.0,2 \mathrm{CH}_{3}\right.$ ethoxy). ${ }^{13} \mathrm{C} \mathrm{NMR}\left(100 \mathrm{MHz}, \mathrm{CDCl}_{3}\right): \delta_{\mathrm{C}} 194.0$ (1C=O ketone), 143.3 (1C aromatic), 136.9 (1C aromatic), 131.9 (1C aromatic), 130.7 (1CH aromatic), 129.8 (1CH aromatic), 127.7 (1CH aromatic), 102.1 (1CH methine), 63.0 (2 $\mathrm{CH}_{2}$ ethoxy), $20.3\left(1 \mathrm{CH}_{3}\right.$ aryl), $19.9\left(1 \mathrm{CH}_{3}\right.$ aryl), $15.4\left(2 \mathrm{CH}_{3}\right.$ ethoxy). HRMS: $\mathrm{m} / z$ calcd for $\mathrm{C}_{12} \mathrm{H}_{15} \mathrm{O}_{2}[\mathrm{M}-\mathrm{OEt}]^{+}$191.10720, found 191.10864. The crystalline material was 3,4-dimethylbenzoic acid (4b); $10 \mathrm{mg}$ (3\%); mp $161-162{ }^{\circ} \mathrm{C}$ (lit. $\left.{ }^{23} 163-165^{\circ} \mathrm{C}\right)$.

Reaction with (E)-2-methylpenta-1,3-diene (2c). The product isolated by FC was 2,2-diethoxy-1-(2,4-dimethylphenyl)ethanone (3c) (colourless oil, 80 mg, 22\%). IR (ATR, $\left.\mathrm{cm}^{-1}\right): 2976(\mathrm{~m}), 2927(\mathrm{w}), 2877(\mathrm{w}), 1681(\mathrm{~m}), 1612$ $(\mathrm{m}), 1564(w), 1445(w), 1379(w), 1324(w), 1293(w), 1271(w), 1237(w), 1111(s), 1058(s), 978(m), 935(w)$, $903(w), 825(w), 763(w), 723(w), 685(w) .{ }^{1} \mathrm{H} N M R\left(400 \mathrm{MHz}, \mathrm{CDCl}_{3}\right): \delta_{\mathrm{H}} 7.97\left(1 \mathrm{H}, \mathrm{d},{ }^{3} \mathrm{~J} 8.8,1 \mathrm{CH}\right.$ aromatic), 7.04-7.10 (2H, m, 2CH aromatic), $5.23\left(1 \mathrm{H}, \mathrm{s}, \mathrm{CH}\right.$ methine), 3.77-3.60 (4H, m, 2 $\mathrm{CH}_{2}$ ethoxy), $2.51\left(3 \mathrm{H}, \mathrm{s}, \mathrm{CH}_{3}\right.$

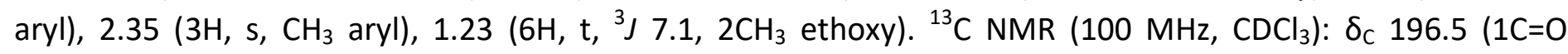
ketone), 142.6 (1C aromatic), 140.5 (1C aromatic), 132.8 (1CH aromatic), 131.1 (1CH aromatic), $130.7(1 \mathrm{CH}$ aromatic), 126.0 (1CH aromatic), $102.3\left(1 \mathrm{CH}\right.$ methine), $62.9\left(2 \mathrm{CH}_{2}\right.$ ethoxy), $21.7\left(1 \mathrm{CH}_{3}\right.$ aryl), $21.5\left(1 \mathrm{CH}_{3}\right.$ aryl), 
15.2 (2 $\mathrm{CH}_{3}$ ethoxy). HRMS: $m / z$ calcd for $\mathrm{C}_{12} \mathrm{H}_{15} \mathrm{O}_{2}[\mathrm{M}-\mathrm{OEt}]^{+} 191.10720$, found 191.09066. The corresponding acid was not obtained.

Reaction of acetate $\mathbf{6}$ with $\mathbf{2 b}$; general procedure. Acetate 6 (3.0 mmol) and 2,3-dimethyl-1,3-butadiene (2b) $(2.47 \mathrm{~g}, 30 \mathrm{mmol})$ were mixed in a round-bottom flask under air and stirred at reflux for $72 \mathrm{hr}$. In every reaction, TLC analysis indicated the formation one product, which was isolated pure by FC, eluting with a 90:10 mixture of hexanes and ethyl acetate. The product was proved to be a derivative of (2-diethoxyacetyl4,5-dimethylphenyl)methyl acetate (7).

Four acetates were prepared, and their structures were elucidated as follows.

(2-Diethoxyacetyl-4,5-dimethylphenyl)methyl acetate (7a). The compound was isolated as a colourless liquid $\left(0.44\right.$ g, 48\%). IR (ATR, cm $\left.{ }^{-1}\right): 2976(\mathrm{~m}), 2882(\mathrm{w}), 1739(\mathrm{~s}), 1693(\mathrm{~m}), 1614(\mathrm{w}), 1558(\mathrm{w}), 1446(\mathrm{w}), 1376(\mathrm{~m})$, $1324(w), 1278(w), 1224(s), 1142(m), 1086(s), 1056(s), 909(m), 879(m), 837(w), 791(w), 743(w), 722(w)$. ${ }^{1} \mathrm{H}$ NMR $\left(400 \mathrm{MHz}, \mathrm{CDCl}_{3}\right): \delta_{\mathrm{H}} 7.91\left(1 \mathrm{H}, \mathrm{s}, \mathrm{CH}\right.$ aromatic), $7.25\left(1 \mathrm{H}, \mathrm{s}, \mathrm{CH}\right.$ aromatic), $5.38\left(2 \mathrm{H}, \mathrm{s}, \mathrm{CH} \mathrm{AcO}^{-}\right.$ methyl), 5,26 (1H, s, $\mathrm{CH}$ methine), 3.78-3.61 (4H, m, $2 \mathrm{CH}_{2}$ ethoxy), $2.31\left(6 \mathrm{H}, \mathrm{s}, 2 \mathrm{CH}_{3} \operatorname{aryl}\right), 2.12\left(3 \mathrm{H}, \mathrm{s}, \mathrm{CH}_{3} \mathrm{Ac}\right)$, $1.24\left(6 \mathrm{H}, \mathrm{t},{ }^{3} \mathrm{~J} 7.1,2 \mathrm{CH}_{3}\right.$ ethoxy). ${ }^{13} \mathrm{C} \mathrm{NMR}\left(100 \mathrm{MHz}, \mathrm{CDCl}_{3}\right): \delta_{\mathrm{C}} 196.8(1 \mathrm{C}=\mathrm{O}$ ketone), $171.3(1 \mathrm{C}=\mathrm{O} \mathrm{AcO}), 142.6$ (2C aromatic), 136.4 (1C aromatic), 136.1 (1C aromatic), 132.7 (1CH aromatic), 130.7 (1CH aromatic), 102.6 (1CH methine), 65.2 ( $1 \mathrm{CH}_{2} \mathrm{AcO}-$ methyl), 63.6 (2 $\mathrm{CH}_{2}$ ethoxy), $21.7\left(1 \mathrm{CH}_{3}\right.$ aryl), $20.8\left(1 \mathrm{CH}_{3}\right.$ aryl), $20.2\left(1 \mathrm{CH}_{3} \mathrm{Ac}\right)$, 15.9 ( $2 \mathrm{CH}_{3}$ ethoxy). HRMS: $\mathrm{m} / z$ calcd for $\mathrm{C}_{15} \mathrm{H}_{19} \mathrm{O}_{4}[\mathrm{M}-\mathrm{OEt}]^{+} 263.12833$, found 263.12711 .

1-(2-Diethoxyacetyl-4,5-dimethylphenyl)ethyl acetate (7b). The compound was isolated as a colourless liquid $(0.46 \mathrm{~g}, 48 \%)$. IR (ATR, cm $\left.{ }^{-1}\right): 2981(w), 2836(w), 2881(w), 1731(\mathrm{~s}), 1681(\mathrm{~s}), 1611(\mathrm{w}), 1557(\mathrm{w}) 1449(\mathrm{w}) 1365$ (m), $1303(\mathrm{~m}), 1273(\mathrm{w}), 1235$ (s), $1192(\mathrm{w}), 1098(\mathrm{~s}), 1069$ (s), 1032 (s), $973(\mathrm{~m}), 937(\mathrm{~m}), 905$ (w), $890(\mathrm{~m}), 851$ (w), $774(w), 746(\mathrm{~m}), 729(\mathrm{w}), 682(\mathrm{~m}) .{ }^{1} \mathrm{H} \mathrm{NMR}\left(500 \mathrm{MHz}, \mathrm{CDCl}_{3}\right): \delta_{\mathrm{H}} 7.81(1 \mathrm{H}, \mathrm{s}, \mathrm{CH}$ aromatic), $7.35(1 \mathrm{H}, \mathrm{s}, \mathrm{CH}$ aromatic), $6.25\left(1 \mathrm{H}, \mathrm{q},{ }^{3} \mathrm{~J} 6.5, \mathrm{CH}\right.$ in $\left.\mathrm{Me}(\mathrm{AcO}) \mathrm{CH}\right), 5,30\left(1 \mathrm{H}, \mathrm{s}, \mathrm{CH}(\mathrm{EtO})_{2}\right.$ methine), 3.82-3.63 $\left(4 \mathrm{H}, \mathrm{m}, 2 \mathrm{CH}_{2}\right.$ ethoxy), $2.33\left(3 \mathrm{H}, \mathrm{s}, \mathrm{CH}_{3}\right.$ aryl), $2.30\left(3 \mathrm{H}, \mathrm{s}, \mathrm{CH}_{3}\right.$ aryl), $2.06\left(3 \mathrm{H}, \mathrm{s}, \mathrm{CH}_{3} \mathrm{Ac}\right), 1.58\left(3 \mathrm{H}, \mathrm{d},{ }^{3} \mathrm{~J} 6.5, \mathrm{CH}_{3} \mathrm{AcO}-\mathrm{ethyl}\right)$, 1.29-1.22 (6H, m, 2 $\mathrm{CH}_{3}$ ethoxy). $\left.{ }^{13} \mathrm{C} \mathrm{NMR} \mathrm{(125} \mathrm{MHz,} \mathrm{CDCl} 3\right): \delta_{\mathrm{C}} 196.8$ (1C=O ketone), $170.0(1 \mathrm{C}=\mathrm{O} \mathrm{AcO}), 142.2$ (1C aromatic), 141.9 (1C aromatic), 135.1 (1C aromatic), 130.9 (1CH aromatic), 130.5 (1C aromatic), $127.3(1 \mathrm{CH}$ aromatic), 101.9 (1CH (EtO) ${ }_{2}$ methine), 69.7 (1CH AcO-ethyl), $62.9\left(1 \mathrm{CH}_{2}\right.$ ethoxy), $62.8\left(1 \mathrm{CH}_{2}\right.$ ethoxy), 22.7 $\left(1 \mathrm{CH}_{3}\right.$ aryl), $21.3\left(1 \mathrm{CH}_{3}\right.$ aryl), $20.2\left(1 \mathrm{CH}_{3} \mathrm{Ac}\right), 15.22\left(1 \mathrm{CH}_{3}\right.$ ethoxy), $15.17\left(1 \mathrm{CH}_{3}\right.$ ethoxy). HRMS: $\mathrm{m} / z$ calcd for $\mathrm{C}_{16} \mathrm{H}_{21} \mathrm{O}_{4}[\mathrm{M}-\mathrm{OEt}]^{+} 277.14398$, found 277.13788 .

1-(2-Diethoxyacetyl-4,5-dimethylphenyl)octyl acetate (7c): The compound was isolated as a colourless liquid $(0.49 \mathrm{~g}, 41 \%)$. IR (ATR, cm $\left.{ }^{-1}\right): 2926(\mathrm{~m}), 2857(\mathrm{w}), 1739(\mathrm{~s}), 1690(\mathrm{~m}), 1612(\mathrm{w}), 1559(\mathrm{w}), 1453(\mathrm{w}), 1370(\mathrm{~m})$, 1300 (w), 1233 (s), 1061 (s), 1021 (s), 888 (w), 839 (w), $724(w), 685(w) .{ }^{1} \mathrm{H}$ NMR (400 MHz, CDCl $): \delta_{H} 7.79$ $\left(1 \mathrm{H}, \mathrm{s}, \mathrm{CH}\right.$ aromatic), $7.27\left(1 \mathrm{H}, \mathrm{s}, \mathrm{CH}\right.$ aromatic), $6.10\left(1 \mathrm{H}, \mathrm{dd},{ }^{3} \mathrm{~J} 5.4,3 \mathrm{~J} 3.5, \mathrm{CH}\right.$ octyl methine), 5,32 (1H, s, CH (EtO) $)_{2}$ methine), 3.80-3.62 (4H, m, 2 $\mathrm{CH}_{2}$ ethoxy), $2.30\left(3 \mathrm{H}, \mathrm{s}, \mathrm{CH}_{3}\right.$ aryl), $2.28\left(3 \mathrm{H}, \mathrm{s}, \mathrm{CH}_{3}\right.$ aryl), $2.05\left(3 \mathrm{H}, \mathrm{s}, \mathrm{CH}_{3}\right.$ Ac), $1.37-1.18\left(18 \mathrm{H}, \mathrm{m}, 2 \mathrm{CH}_{3}\right.$ ethoxy and $6 \mathrm{CH}_{2}$ alkyl), $0.87\left(3 \mathrm{H}, \mathrm{d},{ }^{3} \mathrm{~J} 6.6, \mathrm{CH}_{3}\right.$ octyl). ${ }^{13} \mathrm{C} \mathrm{NMR}\left(125 \mathrm{MHz}, \mathrm{CDCl}_{3}\right)$ : $\delta_{\mathrm{C}} 197.2$ (1C=O ketone), 171.0 (1C=O AcO), 142.41 (1C aromatic), 142.39 (1C aromatic), 135.7 (1C aromatic), 131.6 (1CH aromatic), 131.2 (1C aromatic), 128.1 (1CH aromatic), 102.2 (1CH (EtO) 2 methine), 73.9 (1CH AcOoctyl), 63.5 ( $1 \mathrm{CH}_{2}$ ethoxy), $63.2\left(1 \mathrm{CH}_{2}\right.$ ethoxy), 37.8 ( $1 \mathrm{CH}_{2}$ octy), 32.5 ( $1 \mathrm{CH}_{2}$ octy), $30.0\left(1 \mathrm{CH}_{2}\right.$ octy), $29.9\left(1 \mathrm{CH}_{2}\right.$ octy), 26.8 ( $1 \mathrm{CH}_{2}$ octy), 23.3 ( $1 \mathrm{CH}_{2}$ octy), $21.9\left(1 \mathrm{CH}_{3}\right.$ aryl), $20.9\left(1 \mathrm{CH}_{3}\right.$ aryl), $20.1\left(1 \mathrm{CH}_{3} \mathrm{Ac}\right), 15.9\left(1 \mathrm{CH}_{3}\right.$ ethoxy), 15.8 ( $1 \mathrm{CH}_{3}$ ethoxy), 14.7 ( $1 \mathrm{CH}_{3}$ octy). HRMS: $\mathrm{m} / z$ calcd for $\mathrm{C}_{22} \mathrm{H}_{33} \mathrm{O}_{4}[\mathrm{M}-\mathrm{OEt}]^{+} 361.23788$, found 361.23801.

(2-Diethoxyacetyl-4,5-dimethylphenyl)(phenyl)methyl acetate (7d). The compound was a colourless liquid (1.04 g, 90\%). IR (ATR, cm $\left.{ }^{-1}\right): 2976(w), 2926(w), 1737$ (s), $1686(\mathrm{~m}), 1610(w), 1557(w), 1495(w), 1369(\mathrm{~m})$, $1323(w), 1299(w), 1226$ (s), 1181 (w), $1141(w), 1058$ (s), $1020(s), 965(m), 903(m), 848$ (w), $801(w), 730$ (m), $697(\mathrm{~s}) .{ }^{1} \mathrm{H}$ NMR $\left(400 \mathrm{MHz}, \mathrm{CDCl}_{3}\right): \delta_{\mathrm{H}} 7.84(1 \mathrm{H}, \mathrm{s}, \mathrm{CH}$ aromatic), $7.46(1 \mathrm{H}, \mathrm{s}, \mathrm{CH}$ aromatic), $7.41-7.36(3 \mathrm{H}$, $\mathrm{m}, 3 \mathrm{CH}$ aromatic), 7.30-7.25 $\left(3 \mathrm{H}, \mathrm{m}, 2 \mathrm{CH}\right.$ aromatic and $1 \mathrm{CH} \mathrm{AcO}$-methine), 5,18 (1H, s, $\mathrm{CH}$ (EtO) ${ }_{2}$ methine), 
3.71-3.53 (4H, m, 2 $\mathrm{CH}_{2}$ ethoxy), $2.297\left(3 \mathrm{H}, \mathrm{s}, \mathrm{CH}_{3}\right.$ aryl), $2.290\left(3 \mathrm{H}, \mathrm{s}, \mathrm{CH}_{3} \operatorname{aryl}\right), 2.10\left(3 \mathrm{H}, \mathrm{s}, \mathrm{CH}_{3} \mathrm{Ac}\right), 1.19(6 \mathrm{H}, \mathrm{m}$, $2 \mathrm{CH}_{3}$ ethoxy). ${ }^{13} \mathrm{C} \mathrm{NMR}\left(125 \mathrm{MHz}, \mathrm{CDCl}_{3}\right): \delta_{\mathrm{C}} 196.5$ (1C=O ketone), 169.7 (1C=O AcO), 141.6 (1C aromatic), 140.3 (1C aromatic), 139.1 (1C aromatic), 135.6 (1C aromatic), 131.4 (1CH aromatic), 131.2 (1CH aromatic), 129.0, 128.1, 127.8 and 127.6 (1C aromatic and 5CH aromatic), 102.1 (1CH (EtO) $)_{2}$ methine), $73.9(1 \mathrm{CH}$ (AcO)(Ph)methyl), $62.93\left(1 \mathrm{CH}_{2}\right.$ ethoxy), $62.86\left(1 \mathrm{CH}_{2}\right.$ ethoxy), $37.8\left(1 \mathrm{CH}_{2}\right.$ octy), $32.5\left(1 \mathrm{CH}_{2}\right.$ octy), $30.0\left(1 \mathrm{CH}_{2}\right.$ octy), $29.9\left(1 \mathrm{CH}_{2}\right.$ octy), $26.8\left(1 \mathrm{CH}_{2}\right.$ octy), $23.3\left(1 \mathrm{CH}_{2}\right.$ octy), $21.3\left(1 \mathrm{CH}_{3}\right.$ aryl), $20.3\left(1 \mathrm{CH}_{3}\right.$ aryl), $19.5\left(1 \mathrm{CH}_{3} \mathrm{Ac}\right)$, 15.2 (2 $\mathrm{CH}_{3}$ ethoxy). HRMS: $\mathrm{m} / z$ calcd for $\mathrm{C}_{21} \mathrm{H}_{23} \mathrm{O}_{4}[\mathrm{M}-\mathrm{OEt}]^{+} 339.15963$, found 339.15999.

\section{Acknowledgements}

Financial support from the University of Bergen, the Research Council of Norway, and the Munin Foundation is highly appreciated. Valuable discussions with Professor Miguel Sierra, Compultensae University of Madrid, Spain, are indeed acknowledged. Thanks are also due to Dr. Bjarte Holmelid, University of Bergen, for skilful running of the MS spectra.

\section{Supplementary Material}

The IR spectra and the ${ }^{1} \mathrm{H}$ - and ${ }^{13} \mathrm{C}-\mathrm{NMR}$ spectra of the new compounds are available using the link "Supplementary Material" in the journal contents page.

\section{References}

1. Oppolzer, W. In Comprehensive Organic Synthesis: Selectivity, Strategy \& Efficiency in Modern Organic Chemistry; Trost, B. M.; Fleming, I.; Paquette, L. A. Eds.; Pergamon Press: Oxford, U.K., 1991, 316-400.

2. Fringuelli, F.; Taticchi, A. Dienes in the Diels-Alder Reaction; John Wiley \& Sons: New York, USA, 1990.

3. Fringuelli, F.; Taticchi, A. The Diels-Alder Reaction: Selected Practical Methods; John Wiley \& Sons: New York, USA, 2003.

4. Winkler, J. D. Chem. Rev. 1996, 96, 167. https://doi.org/10.1021/cr950029z

5. Fallis, A. G. Acc. Chem. Res. 1999, 32, 464. https://doi.org/10.1021/ar970120k

6. Fallis, A. G. Can. J. Chem. 1999, 77, 159. https://doi.org/10.1139/v98-233

7. Najera, C.; Sydnes, L. K.; Yus, M. Chem. Rev. 2019, 119, 1110. https://doi.org/10.1021/acs.chemrev.9b00277

8. Sydnes, L. K. Eur. J. Org. Chem.. 2000, 3511. https://doi.org/10.1002/1099-0690(200011)2000:21<3511::AID-EJOC3511>3.0.CO;2-Q

9. Kvernenes, O. H.; Sydnes, L. K. Org. Synth. 2005, 83, 184. https://doi.org/10.15227/orgsyn.083.0184 
10. Sydnes, L. K.; Holmelid, B.; Kvernenes, O. H.; Sandberg, M.; Hodne, M.; Bakstad, E. Tetrahedron 2007, 63, 4144.

https://doi.org/10.1016/i.tet.2007.02.098

11. Cowie, J. M. G. Polymers: Chemistry \& Physics of Modern Materials, $2^{\text {nd }}$ ed; Chapman \& Hall: London, UK, $1991,137$.

12. Petrov, A. A. Zh. Obshch. Khim. 1947, 17, 497; Chem. Abstr. 1948, 42, 4130.

13. Weyerstahl, P.; Marshall-Weyerstahl, H.; Scholz, S. Liebigs Ann. Chem. 1986. 1248. https://doi.org/10.1002/jlac.198619860607

14. Li, W.; Zhou, L.; Zhang, J. Chem. Eur. J. 2016, 22, 1558. https://doi.org/10.1002/chem.201503571

15. Sydnes, L. K.; Holmelid, B.; Sengee, M.; Hanstein, M. J. Org. Chem. 2009, 74, 3430. https://doi.org/10.1021/jo9003986

16. Sydnes, L. K.; Isanov, R.; Sengee, M.; Livi, F. Synth. Commun. 2013, 43, 2898. https://doi.org/10.1080/00397911.2012.748076

17. Heyes, S.; Dobson, C. M. Macromolecules 1992, 25, 3617. https://doi.org/10.1021/ma00040a002

18. Allcock, H.; Silverberg, E. N.; Dudley, G. K.; Pucker, S. R. Macro-molecules 1994, 27, 7550. https://doi.org/10.1021/ma00104a008

19. Steiner, T. Angew. Chem., Int. Ed. 2002, 41, 48. ttps://doi.org/10.1002/1521-3773(20020104)41:1<48::AID-ANIE48>3.0.CO;2-U

20. Takahashi, O.; Kohno, Y.; Nishio, M. Chem. Rev. 2010, 110, 6049. https://doi.org/10.1021/cr100072x

21. Holme, A.; Børve, K. J.; Sæthre, L. J.; Thomas, T. D. J. Phys. Chem. A 2013, 117, 2007. https://doi.org/10.1021/cr100072x

22. Sengee, M.; Sydnes, L. K Synthesis 2011, 3899. https://doi.org/10.1055/s-0031-1289294

23. Melting points and IR/NMR data for 4 available on https://www.sigmaaldrich.com (checked 2020.08.15)- 